\title{
Colecistectomía por laparoscopía: Abordaje con dos incisiones de entrada*
}

\author{
Dr. MARCOS ROCHA G. ${ }^{1}$ \\ 1 Departamento de Gastroenterología y Cirugía Digestiva. Clínica Dávila. Santiago, Chile.
}

\section{Laparoscopic cholecystectomy: Two ports access}

\section{Introducción}

Desde que Philippe Mouret $^{1}$ en Lyon, Francia, en 1987 practicó la primera colecistectomía por laparoscopía, este nuevo método marcó un hito en la cirugía, extendiéndose gradualmente, a otros procedimientos quirúrgicos más allá de los límites de la cirugía abdominal digestiva. Esto apoyó, por otro lado, el nacimiento de un nuevo concepto en muchos procedimientos quirúrgicos, enmarcados en la búsqueda de técnicas mínimamente invasivas, incluyendo endoscópicas, que pudieran tener los beneficios de una mejor calidad en el período postoperatorio, y recuperación del paciente, y que además pudieran repercutir en el costo económico global del mismo.

En este sentido, en patología quirúrgica abdominal, se han realizado recientemente procedimientos endoscópicos y transabdominales mixtos ${ }^{2,3}$ utilizando orificios naturales o la cicatriz umbilical (NOTES, NOTUS), practicándose así colecistectomías con vía de abordaje transluminal, complementadas con un acceso mínimo en pared abdominal ${ }^{2}$. Estas técnicas, sin embargo, están aún en etapa de perfeccionamiento tecnológico y evaluación de sus costos y resultados ${ }^{4-6}$.

El objetivo de la presente comunicación, es presentar una nueva técnica de colecistectomía vía laparoscópica, con utilización de 2 únicas mini-incisiones como puertos de entrada, pero con un costo y aprendizaje similar a la técnica laparoscópica convencional.

\section{Técnica quirúrgica}

Posición de paciente en decúbito dorsal, técnica americana. Posición del cirujano a izquierda del paciente, y de ayudante frente a cirujano, que se podrá cambiar posteriormente a izquierda de este último. Realización de neumoperitoneo de acuerdo a técnica habitual con aguja de Veress por mini-incisión en zona umbilical. Colocación de trocar de $10 \mathrm{~mm}$ para la cámara, en zona umbilical. Posterior a exploración y evaluación de factibilidad de procedimiento, incisión epigástrica y colocación de trocar de $10 \mathrm{~mm}$ bajo visión, e inmediatamente proximal a éste, por misma incisión, colocación de trocar de $5 \mathrm{~mm}$ que saldrá, sin embargo, en sitio diferente al anterior trocar, pero adyacente al mismo, dejando así un puente de fascia entre ambos (Figura 1). Por este trocar de $5 \mathrm{~mm}$ se levantará y movilizará el polo vesicular. Por último, cambio transitorio de la cámara al trocar de $10 \mathrm{~mm}$ en epigastrio, para colocar bajo visión trocar de $5 \mathrm{~mm}$ en zona umbilical, por la misma incisión, pero proximal al trocar de $10 \mathrm{~mm}$ puesto en esta zona, y que saldrá al igual que con trocares de epigastrio, por sitio diferente a trocar de $10 \mathrm{~mm}$ (Figura 2). Por este trocar de $5 \mathrm{~mm}$ se movilizará y traccionará principalmente el bacinete de la vesícula.

Disección similar a técnica clásica laparoscópica, con realización de colecistectomía retrógrada, y disección de triángulo de Calot con electrocoagulación instrumental por trocar epigástrico, en este caso el

*Recibido el 21 de Julio de 2009 y aceptado para publicación el 18 de Agosto de 2009.

Correspondencia: Dr. Marcos Rocha G.

Waterloo 848, Santiago, Chile.

E-mail: marrogue2@gmail.com 


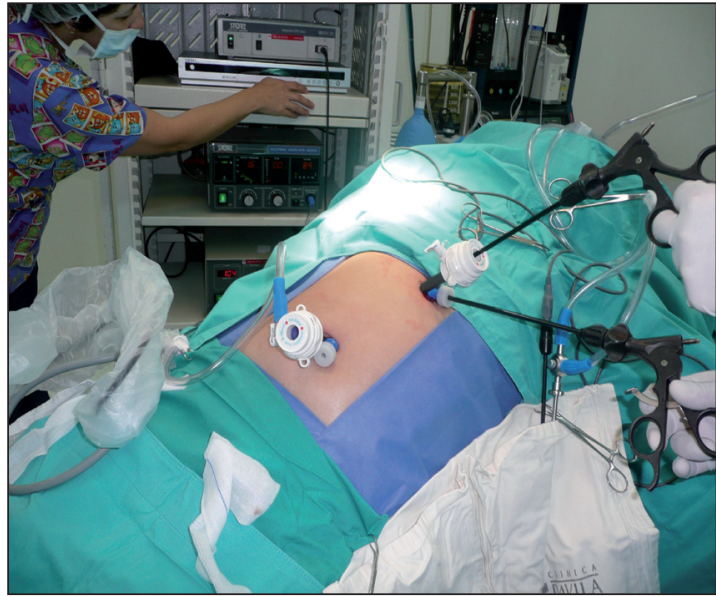

Figura 1. Posición de trocares con dos incisiones de entrada.

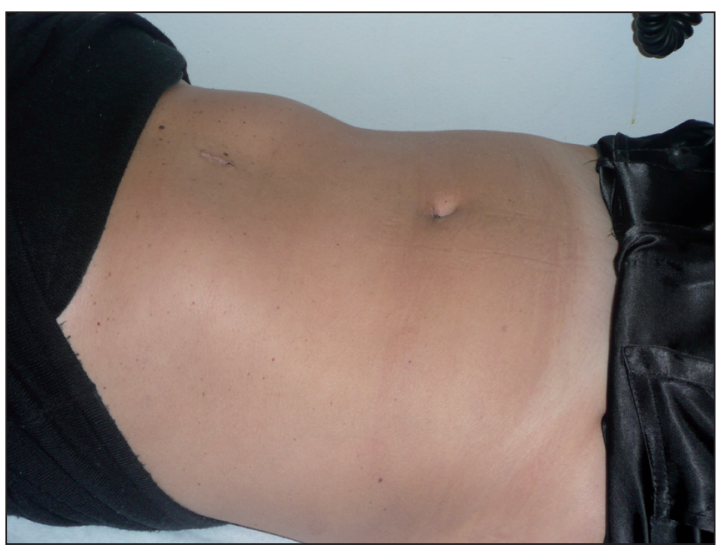

Figura 3. Mini- incisiones en Colecistectomía Laparoscópica con técnica de dos incisiones de entrada.

distal, que será el canal de trabajo para la introducción de instrumentos para disección, colocación de clips hemostáticos, sección de elementos, como en la técnica laparoscópica convencional. Importante señalar con esta técnica, que habitualmente no hay interferencia entre los 2 trocares colocados en epigastrio, dado el buen radio distal de movilidad de los instrumentos de ambos trocares, uno dirigido al polo vesicular, y el otro a proximal de éste, que, sin embargo, se van acercando a medida que avanza la disección. En cambio, en los trocares de la zona umbilical el radio de acción del extremo distal de los instrumentos es menor, por lo que el extremo de la cámara debe seguir prácticamente en todo momento al instrumento introducido por el trocar de $5 \mathrm{~mm}$, moviéndose entonces éstos en forma paralela, lo que no da dificultades en el procedimiento, dado que lo que realice la pinza por el trocar de $5 \mathrm{~mm}$ umbilical

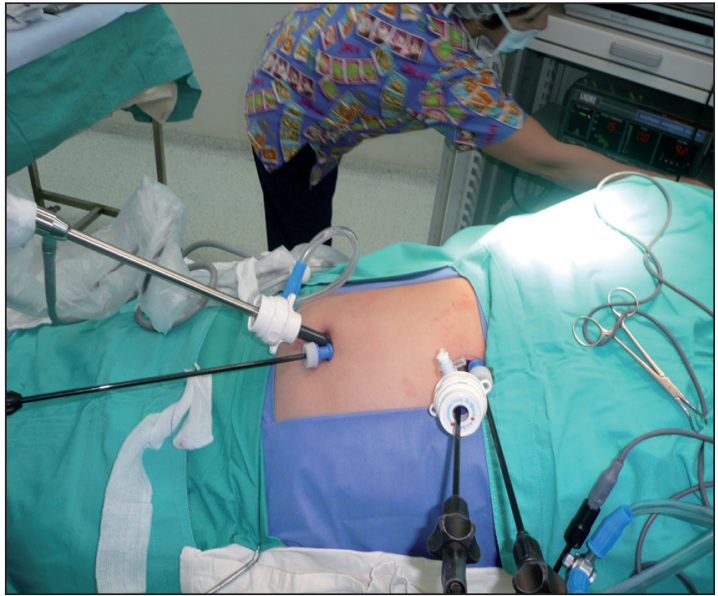

Figura 2. Posición de trocares con dos incisiones de entrada.

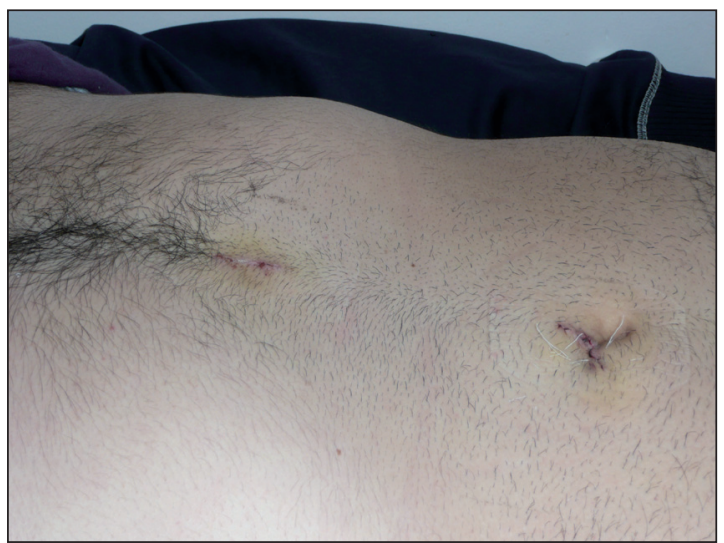

Figura 4. Mini- incisiones en Colecistectomía Laparoscópica con técnica de dos incisiones de entrada.

debe hacerse bajo visión en el conjunto del procedimiento, lográndose esto muy bien con una cámara de 0 grados, por su visión frontal amplia de todo el campo operatorio. Una vez liberada la vesícula, extracción de la misma por incisión epigástrica o umbilical, con o sin ampliación de aponeurosis según características de la misma.

Por último, cierre de rutina de aponeurosis en ambas incisiones con Vicryl 0.

\section{Discusión}

Si bien el concepto de cirugía mínimamente invasiva, aparte de NOTES, ya se ha manifestado en colecistectomía laparoscópica con algunas experiencias, ya sea disminuyendo el diámetro de los instrumentos ${ }^{7,8}$, o el número de trocares ${ }^{9-11}$, impresiona hasta ahora que los métodos descritos han 
sido de valor limitado en la práctica ${ }^{12}$. En el caso de disminución del diámetro de instrumentos esto lleva a dificultades en su manipulación y aprendizaje, al existir problemas en el buen agarre de un fórceps a una pared gruesa vesicular, o en la estabilidad de la sujeción de la pieza que se diseca, con un instrumento frágil que tiende a combarse, por lo que se ha sugerido utilizar estos instrumentos sólo como complementos ${ }^{12}$. Por otra parte, en las técnicas descritas con disminución del número de trocares, se incluyen habitualmente también procedimientos invasivos complementarios en lugares diferentes a la colocación de dichos trocares, que podrían no disminuir, sino mas bien mantener las molestias postoperatorias relacionadas al trauma del acceso a la pared abdominal, comparables a la operación estándar. Estos procedimientos complementarios incluyen, por ejemplo, perforación directa de pared abdominal con instrumentos miniaturizados o agujas, o bien colocación de suturas en la pared vesicular para tracción de la misma, supliendo éstos la falta de otro trocar o instrumento para complementar la separación en la disección ${ }^{10,11,13}$. En 2007, Cuesta ${ }^{14}$ describe el uso de 2 trocares y utilización de elemento miniaturizado como alambre de Kirschner en reborde costal, como complemento en la disección.

En la presente técnica descrita se trabaja sólo con dos mini-incisiones, con las ventajas que esto implica (Figuras 3 y 4), pero al igual que la técnica laparoscópica original, ya sea en su versión americana como francesa, en este método el número de trocares se mantiene, con la consiguiente facilidad y seguridad en la movilización de la vesícula y su disección, comparable entonces a la técnica laparoscópica original.

En este sentido, la técnica no varía mayormente con la convencional ya aprendida y tiene, por lo mismo, una curva de aprendizaje mucho menor, no existiendo otros métodos invasivos o complementarios que pudieran complicar la intervención.

La experiencia en 10 casos, en donde se incluyen 3 cuadros agudos, ha sido satisfactoria en todos los pacientes, sin complicaciones ni conversión a cirugía estándar laparoscópica o laparotómica ${ }^{15}$.

Por otra parte, considerando el costo económico, este nuevo método es comparable a una colecistectomía laparoscópica convencional, sin el agregado de otra instrumentación, existiendo así objetivamente un costo- beneficio mejor a otras técnicas en desarrollo, en donde se incluyen además un aprendizaje y/o un perfeccionamiento tecnológico más complejo para su realización.

Se puede considerar, por último, en la realización de este método, la utilización exclusiva de trócares de $5 \mathrm{~mm}$, con cámara de igual diámetro, y/o de uno de los trocares miniaturizado en epigastrio. Útil es también, para la maniobrabilidad, la utilización como trocares de $5 \mathrm{~mm}$, de aquellos modificados sin paso para gas, pero de diámetro pequeño en su extremo proximal, al menos uno en cada mini-incisión (Figuras 1 y 2).

\section{Referencias}

1. Cervantes C. In Memoriam Dr. Philippe Mouret. Cir Cir 2009; 77: 85-86.

2. Marescaux J, Dallemagne B, Perretta S, Wattiez A, Mutter D, Coumaros D. Surgery without scars: report of transluminal cholecystectomy in a human being. Arch Surg 2007; 142: 823-826.

3. Palanivelu C, Rajan PS, Rangarajan M, Parthasarathi R, Senthilnathan P, Praveenraj P. Transumbilical flexible endoscopic cholecystectomy in humans: first feasibility study using a hybrid technique. Endoscopy 2008; 40: 428-431.

4. Pearl JP, Ponsky JL. Natural orifice translumenal endoscopic surgery: a critical review. J Gastrointest Surg 2008; 12: 1293-1300.

5. Sodergren MH, Clark J, Athanasiou T, Teare J, Yang GZ, Darzi A. Natural orifice translumenal endoscopic surgery: critical appraisal of applications in clinical practice. Surg Endosc 2009; 23: 680-687.

6. Ponsky LE, Poulouse BK, Pearl J, Ponsky JL. Natural Orifice Translumenal Endoscopic Surgery: Myth or Reality? J Endourol 2009 May 7. Epub ahead of print.

7. Gagner M, Garcia-Ruiz A. Technical aspects of minimally invasive abdominal surgery performed with needlescopic instruments. Surg Laparosc Endosc 1988; 8: 171-179.

8. Reardon PR, Kamelgard JI, Applebaum B, Rossman L, Brunicardi FC. Feasibility of laparoscopic cholecystectomy with miniaturized instrumentation in 50 consecutive cases. World J Surg 1999; 23: 128-132.

9. Cerci C, Tarhan OR, Barut I, Bülbül M. Threee-port versus four-port laparoscopic Cholecystectomy. Hepatogastroenterology 2007; 54: 15-16.

10. Mori T, Ikeda Y, OkamotoK, Sakata K, Ideguchi K, Nakagawa K, et al. A new technique for two-trocar laparoscopic cholecystectomy. Surg Endosc 2002: 16: 589-591.

11. Lomanto D, De Angelis L, Ceci V, Dalsasso G, So J, Frattaroli FM, et al. Two-trocar laparoscopic cholecystectomy: a reproducible technique. Surg Laparosc Endosc Percutan Tech 2001; 11: 248-251.

12. Kagaya T. Laparoscopic cholecystectomy via two port, using the "Twing-Port" System. J Hepatobiliary Pancreat Surg 2001; 8: 76-80.

13. Ramachandran CS, Arora V. Two-port laparoscopic cholecystectomy: an innovative new method for gallblader removal. J Laparoendosc Adv Surg Tech A 1998; 8: 303-308. 
14. Cuesta MA, Berenda F, Veenhof AA. The "invisible cholecystectomy": A transumbilical laparoscopic operation without a scar. Surg Endosc 2008; 22: 1211-1213.

15. Rocha M. Colecistectomía por laparoscopía: Vía de abordaje con dos puertos. Sesión Foro Quirúrgico. Cirugía y Tecnología . $53^{\circ}$ Congreso Anual Capítulo Chileno American College of Surgeons. Santiago, Chile. 7 de Mayo 2009. 